\title{
Gaps in the Continuum of HIV Care: Long Pretreatment Waiting Time between HIV Diagnosis and Antiretroviral Therapy Initiation Leads to Poor Treatment Adherence and Outcomes
}

\author{
Shu Su, ${ }^{1}$ Shifu Li, ${ }^{2}$ Shunxiang Li, ${ }^{2}$ Liangmin Gao, ${ }^{2,3}$ Ying Cai, ${ }^{2}$ Jincui Fu, ${ }^{2}$ Chunyuan Guo, ${ }^{2}$ \\ Jun Jing, ${ }^{3}$ Liang Chen, ${ }^{2}$ Limin Mao, ${ }^{4}$ Feng Cheng, ${ }^{3,5}$ and Lei Zhang ${ }^{1,3,6}$ \\ ${ }^{1}$ School of Public Health and Preventive Medicine, Faculty of Medicine, Nursing and Health Sciences, Monash University, \\ Melbourne, VIC, Australia \\ ${ }^{2}$ Division of HIV/AIDS and STI Control, Centers for Disease Control and Prevention, Yuxi Prefecture, Yunnan, China \\ ${ }^{3}$ Research Center for Public Health, School of Medicine, Tsinghua University, Beijing, China \\ ${ }^{4}$ Center for Social Research in Health, Faculty of Arts and Social Science at the University of New South Wales, Sydney, \\ NSW, Australia \\ ${ }^{5}$ Beijing Key Laboratory of Indoor Air Quality and Evaluation and Control, Tsinghua University, Beijing, China \\ ${ }^{6}$ Melbourne Sexual Health Centre, Alfred Health, Melbourne, VIC, Australia \\ Correspondence should be addressed to Feng Cheng; feng.cheng@biomed.tsinghua.edu.cn and \\ Lei Zhang; lzhang@kirby.unsw.edu.au
}

Received 31 August 2016; Revised 25 November 2016; Accepted 7 December 2016

Academic Editor: Valdilea Veloso

Copyright (C) $2016 \mathrm{Shu}$ Su et al. This is an open access article distributed under the Creative Commons Attribution License, which permits unrestricted use, distribution, and reproduction in any medium, provided the original work is properly cited.

\begin{abstract}
Background. Criteria for antiretroviral treatment (ART) were adjusted to enable early HIV treatment for people living HIV/AIDS (PLHIV) in China in recent years. This study aims to determine how pretreatment waiting time after HIV confirmation affects subsequent adherence and outcomes over the course of treatment. Methods. A retrospective observational cohort study was conducted using treatment data from PLHIV in Yuxi, China, between January 2004 and December 2015. Results. Of 1,663 participants, 348 were delayed testers and mostly initiated treatment within 28 days. In comparison, 1,315 were nondelayed testers and the median pretreatment waiting time was 599 days, but it significantly declined over the study period. Pretreatment CD4 T-cell count drop (every 100 cells $/ \mathrm{mm}^{3}$ ) contributed slowly in CD4 recovery after treatment initiation $(8 \%$ less, $P<0.01)$ and increased the risk of poor treatment adherence by $15 \%(A R R=1.15,1.08-1.25)$. Every 100 days of extensive pretreatment waiting time increased rates of loss to follow-up by $20 \%$ (ARR $=1.20,1.07-1.29)$ and mortality rate by $11 \%$ (ARR $=1.11,1.06-1.21$ ), based on multivariable Cox regression. Conclusion. Long pretreatment waiting time in PLHIV can lead to higher risk of poor treatment adherence and HIV-related mortality. Current treatment guidelines should be updated to provide ART promptly.
\end{abstract}

\section{Background}

To date, antiretroviral treatment (ART) is the most effective way to reduce HIV-related mortality among people living with HIV (PLHIV) and prevent further transmission of the virus $[1,2]$. In 2014, the Joint United Nations Programme on HIV and AIDS (UNAIDS) announced an ambitious "90-9090 " goal, in which, by $2020,90 \%$ of HIV-infected individuals would be diagnosed, $90 \%$ would receive ART treatment after diagnosis, and $90 \%$ would achieve viral suppression as a result of effective treatment $[3,4]$. Treatment outcomes of ART may be influenced by multiple factors, but the key one is the timeliness of treatment initiation $[5,6]$, which relies on the early diagnosis of the infection [7, 8]. In 2015, WHO announced its latest treatment guidelines which recommend PLHIV to initiate ART regardless of CD4 level soonest after diagnosis [9]. Developed countries in Europe have already implemented the WHO recommendations [10]. US panels 
also recommended that all individuals with detectable viral loads should receive ART regardless of their CD4+ T-cell counts in 2016 [11]. There is an increasing consensus that ART should be provided to PLHIV as soon as they are diagnosed if resources are available.

The Chinese treatment threshold is synchronously updated with WHO recommendations. In 2008, treatment threshold was improved from the 2002 level of 200 cells $/ \mathrm{mm}^{3}$ to 350 cells $/ \mathrm{mm}^{3}$ [12]. In 2014, the threshold was further increased 500 cells $/ \mathrm{mm}^{3}$ [13]. Currently, the Chinese National Centre for Disease Control and Prevention (CDC) is considering removing CD4 requirements completely to allow patients to initiate treatment at any time after HIV diagnosis. However, given that most PLHIV were diagnosed with a low CD4+ T-cell count [14], removing the treatment threshold may not have a real impact on improving ART coverage. Besides, the treatment resources were not distributed evenly in China, in resource-limited areas, treatment has to be prioritised to PLHIV diagnosed with a low CD4 count [15]. By far no strong evidence in China proves ART with removing CD4 threshold is effective to help the various population in China. Thus, despite updates in treatment guidelines nowadays, many PLHIV patients still experience long waiting time before their CD4+ T-cell counts fall below the threshold level to be qualified for treatment. It is unclear whether these patients, despite linked to HIV care, can benefit from ART to the full extent if they delay their treatment.

Yuxi, with 2.3 million residents, is the third-largest city in Yunnan province in Southwest China. The city is located 100 kilometres south of Kunming, the capital of Yunnan Prefecture, and approximately 300 kilometres north of Vietnam. Its proximity to the traditional drug trafficking route attributes to a size population of injecting drug users (IDUs) [16], resulting in Yuxi having one of the highest HIV rates in China [17]. ART was substantially scaled up in Yuxi in 2004 and provided to over 2,741 PLHIV since then. This study aimed to investigate whether the time elapsed between HIV diagnosis and ART initiation and the variation in CD4+ T-cell level prior to treatment initiation may have strong implications to the subsequent treatment effectiveness.

\section{Methods}

2.1. Data Sources. Data was extracted retrospectively from the treatment and epidemiology databases of the Yuxi CDC. Both databases were maintained according to the requirements of China CDC. The treatment database contained information on all PLHIV who began ART treatment in Yuxi between 2004 and 2015. The epidemiology database contains the results of CD4+ T-cell counts tests taken before ART treatment of patients diagnosed in Yuxi between 1996 and 2015. For the study, each patient's name and another identifiable information in the databases were replaced by a unique code, which was used to link the information in both databases about each subject, enabling the study to collect data on each patient from both before and after ART initiation. The study was approved by Monash University Research Ethics Committee (Project number 0651).
2.2. Inclusion Criteria. The study included participants within the study jurisdiction who were 18 years old or older at the time of ART initiation during the study period, which was between 1 January 2004 and 31 December 2015. The study excluded patients whose data could only be found in one of the epidemiology and treatment databases or who died from any causes other than an HIV-related one (like accidents, suicide, and others) during the study period. Matching the two databases ensures the completeness of indicators for subsequent analysis.

2.3. Baseline Variables. The study collected patients' demographic characteristics at ART initiation, including age, gender, body mass index (BMI), and marital status. Data regarding the date of HIV diagnosis, HIV transmission route, initial CD4 tests, and ART initiation (including CD4+ Tcell counts, WHO HIV clinical stage, and complications) were collected from each patient's initial clinical assessment. Included patients were categorised into two groups by their detention status: (1) delayed testers and (2) nondelayed testers. According to the international criteria, delayed testers were defined as those with CD4+ T-cell counts < 200 cells $/ \mathrm{mm}^{3}$, who were categorised as being in the WHO clinical stage IV, within 360 days after confirmation of HIV-positive status [18]. After categorising the patients as delayed or nondelayed, the two groups were compared for the following: demographic characteristics, baseline variables, and three important indicators. Specifically, these indicators include the days between HIV diagnosis and ART initiation, which was used to demonstrate how long a patient should wait for treatment according to the previous criteria and the elapsed time from HIV diagnosis to CD4 testing, which represents how quickly a patients were connected to HIV care; in the nondelayed group, the various values and rates of decrease CD4+ T-cell counts were recorded as an independent variable, which has reflected the function of immune system of patients before ART. The declined value was stratified into five groups by every 100 cells $/ \mathrm{mm}^{3}$ for the purpose of analyzing the relationship between the pretreatment CD4+ T-cell counts decline difference and treatment outcomes (Figure 1).

2.4. Outcome Variables. There are three outcome variables in the study: (1) immunological response (CD4+ T-cell count increase) during the first 6-12 months after ART initiation, (2) subsequent HIV-related mortality, and (3) loss to followup (Figure 1).

2.5. Statistical Analyses. The study conducted both descriptive and inferential statistical analyses. Median and interquartile ranges (IQR) were used to summarise numerical variables, whereas frequency times and percentages were used to summarise categorical variables. The temporal trend of the duration of diagnosis and treatment and the duration between diagnosis and first CD4+ T-cell testing was calculated by linear trend test. A linear trend test was also used to investigate the CD4+ T-cell counts variation value associated with immunological response in the first 6-12 


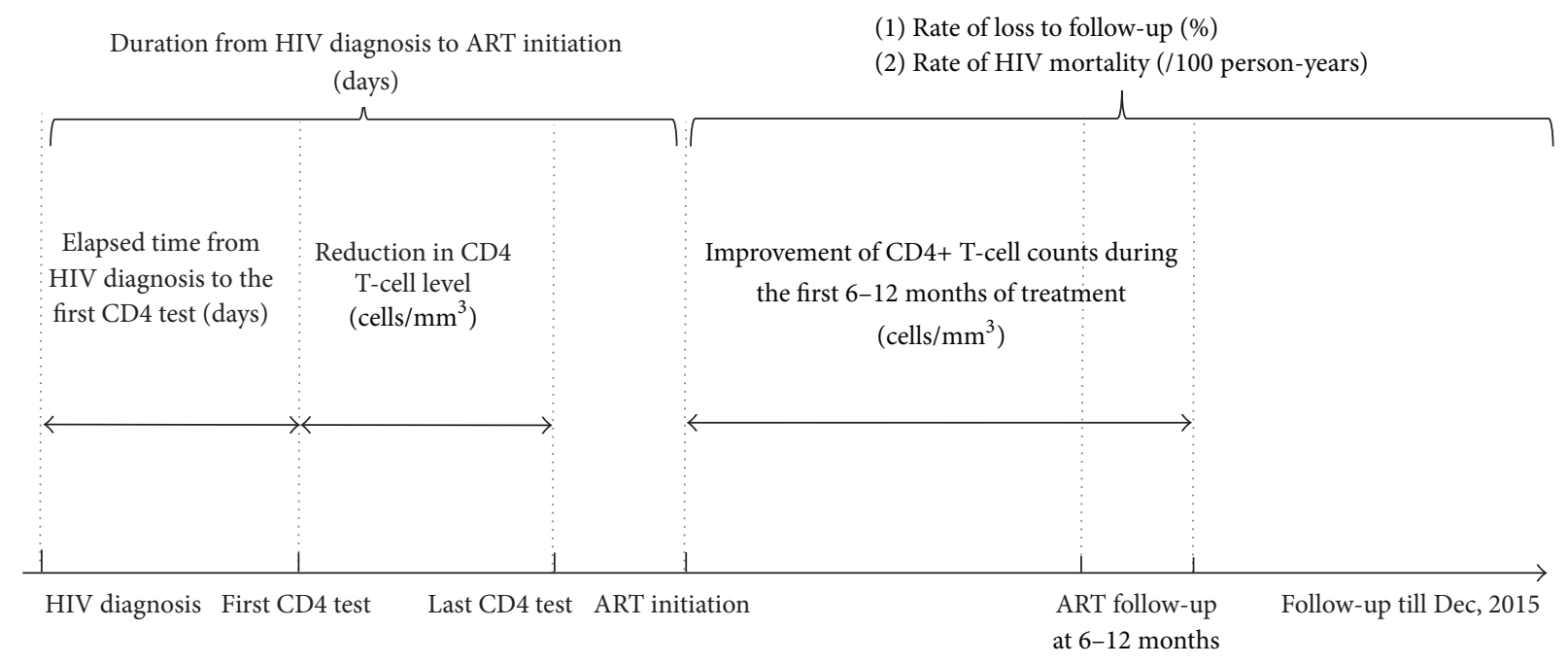

FIGURE 1: Illustration of key indicators used in this study.

months of treatment. Adjusted relative risks (ARRs) accompanied by $95 \%$ confidence intervals (CIs) were calculated. A multivariable Cox regression was used to identify key factors related to mortality and adherence rate. The mortality rate was calculated as the number of recorded deaths divided by the total number of person-years (PYs) from the initiation of treatment to the end of follow-up. The rate of loss to follow-up was calculated as the number of participants with recorded ART discontinuation divided by the total number of PYs in the same period. Variables with a $P$ value $<0.2$ in the univariate analysis were included in the multivariate analyses. Collinearity was checked by evaluating the impact factors of all the indicator variables in the multivariate analyses. Data management and analysis were conducted using Statistical Analysis System 9.4, with $P$ values set at $<0.05$ throughout. Missing data were included as "unknown."

\section{Results}

3.1. Baseline of Participant Characteristics. A total of 1,953 PLHIV adults enrolled in this study over the period from January 2004 to December 2015. We excluded 155 patients who were only in the epidemiology database, 109 patients were only in the treatment database, and 26 patients died of non-HIV-related causes (19 from nondelayed testers and 7 from delayed testers). The remaining 1,663 PLHIV were finally included. Among them, 348 (20.9\%) were delayed testers while $1,315(79.1 \%)$ were nondelayed testers. The median age of participants was 36 years (IQR 30-45), and the median body measure index (BMI) was $21.3 \mathrm{~kg} / \mathrm{m}^{2}$ (IQR $19.3-$ 23.1). Delayed testers were significantly elder (42 years [3450] versus 35 years [30-43], $P<0.01$ ) and with lower BMI $\left(19.1 \mathrm{~kg} / \mathrm{m}^{2}\right.$ [17.4-20.8] versus $21.4 \mathrm{~kg} / \mathrm{m}^{2}$ [19.5-23.2], $P<$ $0.01)$ than nondelayed population. People who acquired HIV by sharing of injection equipment $(39,11.1 \%)$ have a lower proportion among nondelayed testers $(313,23.8 \%)$ than delayed testers $(P<0.01)$. Among PLHIV with known hepatitis infection status, patients in the nondelayed group have a higher HCV coinfection rate than the delayed group (225, 24.7\% versus $28,11.7 \%, P<0.05)$, while the HBV coinfection prevalence did not differ between the two groups $(64,6.3 \%$ versus $18,6.8 \%, P=0.76)$ (Table 1$)$. At the diagnosed stage, HCV prevalence in the nondelayed group was $20.2 \%(88 / 435)$ and $12.4 \%(21 / 169)$ in delayed testers. HBV prevalence in two groups was 5.7\% (25/435) and 5.9\% (10/169), respectively.

3.2. Pretreatment Waiting Time and Disease Progression. It was found that nondelayed testers had to wait much longer to receive ART after HIV diagnosis than the delayed testers (599, [interquartile range, IQR $=54-1740$ ] versus 28 [15-56] days) although the waiting time from diagnosis to the first CD4 test was similar (13 [7-22] days versus 16 [7-56.5] days, resp.). However, temporally, waiting time for nondelayed testers has significantly declined during 2004-2015. The days between HIV infection confirmation and treatment declined from 1706 (IQR: $507-3,123$ ) in 2004-2006 to 762 days IQR (243$1,611)$ in 2007-2009 then 588 days IQR $(69-1,548)$ in 20102012 and 447 days IQR $(37-1,639)$ in 2013-2015 (linear trend test, $P<0.05)$, respectively. Similarly, the elapsed time from diagnosis to first CD4 test, which represented the time taken to link to HIV care, declined from 99 days (56-117) to 11 days (4-23) over the same period (Figures 2(a) and 2(b)).

3.3. Predictors of Treatment Effects and Adherence. The pretreatment variation of the CD4 level and waiting time determined long-term ART outcomes. We found that longterm ART effects and pretreatment drop difference (every 100 cells $/ \mathrm{mm}^{3}$ ) were associated with $8 \%$ reduction in CD4 recovery after treatment initiation (linear trend test, $P<$ 0.01) (Figure 3). Of 1,663 participants, 169 patients died due to HIV-related diseases over the treatment follow-up. As for 
TABLE 1: Key demographic characteristics and hepatitis coinfection prevalence rates of participants in Yuxi, China, stratified by delayed testers and nondelayed testers.

\begin{tabular}{|c|c|c|c|c|}
\hline & Total & Delayed testers & Nondelayed testers & $P$ value \\
\hline Demographic characteristics & $1663(100 \%)$ & $348(20.9 \%)$ & $1315(79.1 \%)$ & \\
\hline Gender & & & & 0.02 \\
\hline Female & $661(39.7 \%)$ & $120(18.2 \%)$ & $541(81.8 \%)$ & \\
\hline Male & $1002(60.3 \%)$ & $228(22.8 \%)$ & $774(77.2 \%)$ & \\
\hline Age (IQR) & $36(30-45)$ & $42(34-50)$ & $35(30-43)$ & $<0.01$ \\
\hline BMI (IQR) & $21.3(19.3-23.1)$ & $19.1(17.4-20.8)$ & $21.4(19.5-23.2)$ & $<0.01$ \\
\hline Marriage status & & & & 0.04 \\
\hline Married & $1156(69.5 \%)$ & $263(22.8 \%)$ & $893(77.2 \%)$ & \\
\hline Single & $261(15.7 \%)$ & $41(15.7 \%)$ & $220(84.3 \%)$ & \\
\hline Divorced & $181(10.9 \%)$ & $36(19.9 \%)$ & $145(80.1 \%)$ & \\
\hline Widowed & $62(3.7 \%)$ & $8(12.9 \%)$ & $54(87.1 \%)$ & \\
\hline Unknown & $3(0.2 \%)$ & $0(0 \%)$ & $3(100 \%)$ & \\
\hline Transmission route & & & & $<0.01$ \\
\hline Heterosexuals & $1158(69.6 \%)$ & $265(22.9 \%)$ & $893(77.1 \%)$ & \\
\hline IDU & $352(21.2 \%)$ & $39(11.1 \%)$ & $313(88.9 \%)$ & \\
\hline Homosexuals & $46(2.8 \%)$ & $9(19.6 \%)$ & $37(80.4 \%)$ & \\
\hline FPD & $9(0.5 \%)$ & $2(22.2 \%)$ & $7(77.8 \%)$ & \\
\hline Unknown & $98(5.9 \%)$ & $33(33.7 \%)$ & $65(66.3 \%)$ & \\
\hline ART initiation year & & & & 0.04 \\
\hline 2004-2006 & $19(1.1 \%)$ & $7(36.8 \%)$ & $12(63.2 \%)$ & \\
\hline $2007-2009$ & $218(13.1 \%)$ & $53(24.3 \%)$ & $165(75.7 \%)$ & \\
\hline 2010-2012 & $602(36.2 \%)$ & $139(23.1 \%)$ & $463(76.9 \%)$ & \\
\hline $2013-2015$ & $796(47.9 \%)$ & $149(18.7 \%)$ & $647(81.3 \%)$ & \\
\hline Unknown & $28(1.7 \%)$ & $0(0 \%)$ & $28(100 \%)$ & \\
\hline CD4 counts at ART initiation & & & & $<0.01$ \\
\hline $0-200$ & $513(30.8 \%)$ & $339(66.1 \%)$ & $174(33.9 \%)$ & \\
\hline $200-350$ & $641(38.5 \%)$ & $8(0.4 \%)$ & $633(96.4 \%)$ & \\
\hline $350-500$ & $258(15.5 \%)$ & $1(0.1 \%)$ & $257(99.9 \%)$ & \\
\hline$>500$ & $251(15.1 \%)$ & $0(0 \%)$ & $251(100 \%)$ & \\
\hline \multicolumn{5}{|l|}{ Hepatitis coinfection } \\
\hline HCV coinfection & & & & $<0.001$ \\
\hline Positive & $253(15.2 \%)$ & $28(11.7 \%)$ & $225(24.7 \%)$ & \\
\hline Negative & $897(53.9 \%)$ & $212(88.3 \%)$ & $685(76.3 \%)$ & \\
\hline HBV coinfection & & & & 0.76 \\
\hline Positive & $82(4.9 \%)$ & $18(6.8 \%)$ & $64(6.3 \%)$ & \\
\hline Negative & $1193(71.7 \%)$ & $245(93.2 \%)$ & $948(93.7 \%)$ & \\
\hline
\end{tabular}

elevating mortality, every 100 days of extensive pretreatment waiting time increased HIV-mortality rate by $11 \%$ during the following course of ART (ARR = 1.11, 1.06-1.21). However, pretreatment CD4 decline did not affect subsequent mortality. Other independently associated factors of mortality included being older $(\mathrm{ARR}=1.07,1.04-1.09)$, initiated ART in 2007-2009 $(\mathrm{ARR}=2.05,1.26-4.35$ versus patients started treatment in 2013-2015), and HIV infected by drug injection $(\mathrm{ARR}=2.88,1.34-6.17)$ (Table 2).

Among all the alive patients, 68 (4.6\%) have discontinued treatment during the study period. Based on the multivariable Cox regression model, every drop of 100 cells $/ \mathrm{mm}^{3}$ in CD 4 level before treatment could increase the risk of nonadherence by $15 \%(A R R=1.15,1.08-1.25)$. Every 100 days of extensive pretreatment waiting time increased rates of loss to follow-up by $20 \%(\mathrm{ARR}=1.20,1.07-1.29)$. Delayed testers $(\mathrm{ARR}=1.49,1.30-1.72)$ and aging $(\mathrm{ARR}=1.07,1.04-1.09)$ were associated with elevated risks of loss to follow-up as well. In terms of transmission route, IDU has suboptimal performance compared with heterosexuals. The risk of loss to follow-up was increased to 1.53-fold 95\% CI (1.11-2.11) among IDU (Table 2).

\section{Discussion}

This study demonstrated a significant declining trend in the number of delayed testers, the duration of HIV diagnosis and treatment, and the duration between diagnosis and the 

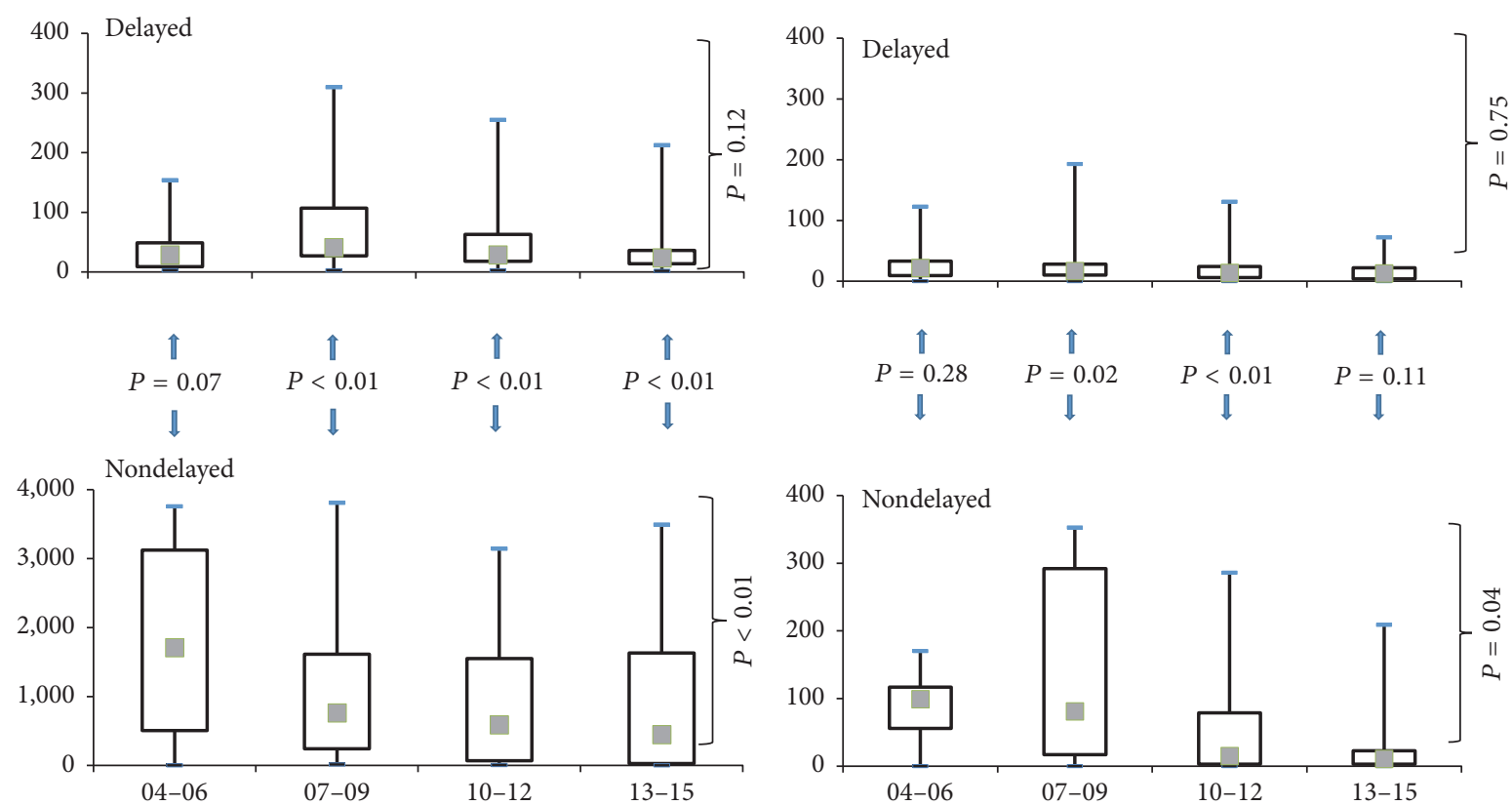

(a)

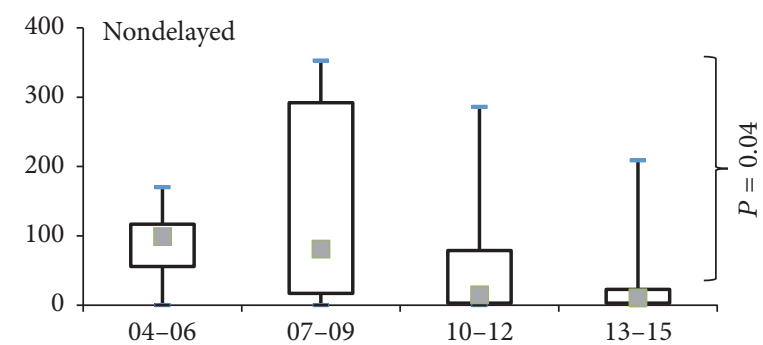

(b)

Figure 2: (a) Days between HIV-positive and ART initiation (delayed and nondelayed). (b) Days between HIV-positive and first CD4 testing date (delayed and nondelayed).

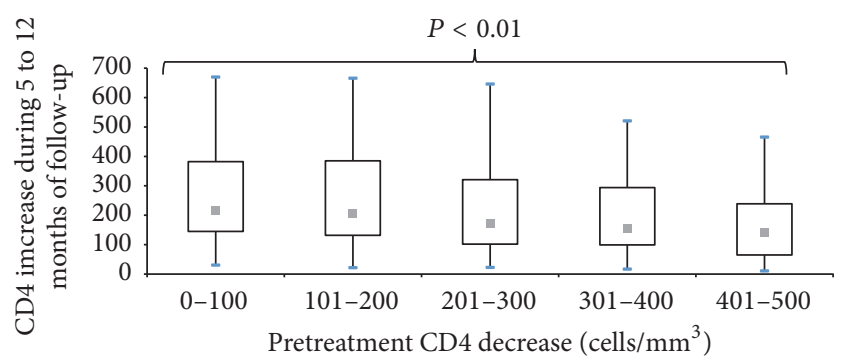

FIGURE 3: The association between CD4+ T-cell counts decrease value before ART initiation and CD4+ T-cell counts increase during first six to twelve months after treatment initiation.

first CD4 test date, consistent with the rising CD4 treatment threshold in the treatment guidelines during the study period. The pretreatment elapsed time in the delayed-tester group was remained unchanged in the same period, indicating HIV care has been provided promptly to PLHIV with very low CD4 T-cell counts or those clinically viable. Pretreatment waiting time and $\mathrm{CD} 4$ decline are important determining factors of subsequent ART adherence, HIV-related mortality, and immune response.

The duration between HIV diagnosis and first CD4+ Tcell counts testing is similar between delayed testers and nondelayed testers. This suggests that most PLHIV enter HIV care quickly within two weeks of diagnosis. Thus, it illustrates that even if nondelayed patients expect to start antiretroviral therapy as soon as the delayed testers, the Chinese treatment program may still be able to cover them sufficiently. In contrast, the duration of HIV diagnosis to first CD4+ T-cell counts test in the nondelayed testing group was shortened gradually, which echoes the enactment of the "Four Frees and One Care" policy in China in 2004 and the scale-up of ART program that improves the linkage of diagnosed patients to care [19].

In contrast to the delayed testing group, the majority of the nondelayed testing group have to wait over a year to initiate ART according the treatment guidelines' standards. Although the treatment threshold has gradually increased over the years $\left(200\right.$ cells $/ \mathrm{mm}^{3}$ in 2002, 350 in 2008, and 500 in 2014) [20], patients in the nondelayed group often have to wait significantly longer than the delayed testing group to be qualified for treatment. Our finding indicates that the wait leads to the potentially higher risk of HIV-related mortality subsequently, due to two main weaknesses. First, patients may continue their risk behavioural pattern in the absence of suitable intervention after diagnosis, which accelerate HIV disease progression or being coinfected by other bloodborne and sexually transmitted infections. Previous studies confirmed that ART reduces their high-risk behaviour as a clinical health care can target and monitor patients in a routine manner [21,22]. Given that patient in the nondelayed group has a higher proportion of IDUs (23\%) than that of the delayed group (11\%), HCV infection is therefore likely more common in this group at ART initiation. It is well known that HIV coinfection would complicate the treatment and increased treatment failure risk leading to high mortality [23-25]. Furthermore, during the pretreatment period, HIV-positive patients with high CD4 counts may regard themselves as the health and neglect medical advice [26]. In contrast, patients who have received correct knowledge about ART and would initiate therapy earlier after diagnosis would 
TABLe 2: Predictors of loss to follow-up and HIV-related mortality over the whole study period among eligible participants in the ART observational database of the Yuxi District.

\begin{tabular}{|c|c|c|c|c|}
\hline & \multicolumn{2}{|c|}{ Loss to follow-up } & \multicolumn{2}{|c|}{ Mortality } \\
\hline & $\begin{array}{c}\text { Cases/participant, } \\
\text { person-years }\end{array}$ & ARR, $P$ value & $\begin{array}{c}\text { Cases/participant, } \\
\text { person-years }\end{array}$ & ARR, $P$ value \\
\hline \multicolumn{5}{|l|}{ Delayed status } \\
\hline Nondelayed testers & $56 / 1212,3869$ & Ref. & $103 / 1315,3995$ & Ref. \\
\hline Delayed testers & $12 / 282,1129$ & $\begin{aligned} 1.49 & (1.30-1.72) \\
& <0.01^{*}\end{aligned}$ & $66 / 348,1243$ & $\begin{array}{c}1.43(0.68-2.26) \\
0.48\end{array}$ \\
\hline \multicolumn{5}{|l|}{ Gender } \\
\hline Female & $18 / 623,1831$ & Ref. & $38 / 661,2038$ & Ref. \\
\hline Male & $50 / 871,3167$ & $\begin{array}{l}1.06(0.95-1.19) \\
0.29\end{array}$ & $131 / 1002,3200$ & $\begin{array}{c}1.44(0.68-3.06), \\
0.34\end{array}$ \\
\hline Age & 68/1494, 4998 & $\begin{array}{c}1.01(1.00-1.01) \\
<0.01^{*}\end{array}$ & $169 / 1663,5238$ & $\begin{aligned} & 1.07 \\
& (1.04-1.09) \\
& <0.01^{*}\end{aligned}$ \\
\hline \multicolumn{5}{|l|}{ Transmission route } \\
\hline Heterosexuals & $37 / 1073,3226$ & Ref. & $85 / 1158,3341$ & Ref. \\
\hline IDU & $27 / 282,1294$ & $\begin{array}{l}1.53(1.11-2.11) \\
<0.01^{*}\end{array}$ & $70 / 352,1368$ & $\begin{aligned} 2.88 & (1.34-6.17) \\
& <0.01^{*}\end{aligned}$ \\
\hline FPD & $0 / 9,24$ & - & $0 / 9,24$ & 0 \\
\hline MSM & $0 / 44,99$ & - & $2 / 46,100$ & $\begin{array}{c}0.54(0.07-4.40) \\
0.57\end{array}$ \\
\hline Unknown & 4/86, 355 & $\begin{array}{l}1.23(0.83-1.47) \\
0.19\end{array}$ & $12 / 98,405$ & $\begin{array}{c}2.11(0.87-2.81) \\
0.33\end{array}$ \\
\hline $\begin{array}{l}\text { Pretreatment waiting time (every } \\
100 \text { days) in nondelayed }\end{array}$ & $56 / 1212,3869$ & $\begin{array}{c}1.20(1.07-1.29) \\
<0.01^{*}\end{array}$ & $103 / 1315,3995$ & $\begin{array}{l}1.11(1.06-1.21) \\
<0.01^{*}\end{array}$ \\
\hline $\begin{array}{l}\text { Pretreatment CD } 4 \text { decline (every } \\
100 \text { cells } / \mathrm{mm}^{3} \text { ) in nondelayed }\end{array}$ & 56/1212, 3869 & $\begin{array}{c}1.15(1.08-1.25) \\
<0.01^{*}\end{array}$ & 103/1315, 3995 & $\begin{array}{c}1.10(0.96-1.16) \\
0.78\end{array}$ \\
\hline \multicolumn{5}{|l|}{ ART initiation year } \\
\hline 2013-2015 & $32 / 754,1590$ & Ref. & $42 / 796,1636$ & Ref. \\
\hline $2010-2012$ & 22/521, 1936 & $\begin{array}{c}0.80(0.67-1.65) \\
0.53\end{array}$ & $69 / 590,1981$ & $\begin{array}{c}1.32(0.61-2.90) \\
0.48\end{array}$ \\
\hline $2007-2009$ & $14 / 169,1089$ & $\begin{array}{c}0.69(0.38-1.68) \\
0.54\end{array}$ & $49 / 218,1205$ & $\begin{array}{l}2.05(1.26-4.35) \\
0.01^{*}\end{array}$ \\
\hline 2004-2006 & $0 / 15,166$ & - & $4 / 19,190$ & $\begin{array}{c}0.92(0.76-8.13) \\
0.35\end{array}$ \\
\hline Unknown & $0 / 23,217$ & - & $5 / 28,226$ & $\begin{array}{c}0.98(0.78-6.25) \\
0.31 \\
\end{array}$ \\
\hline
\end{tabular}

${ }^{*}$ Result is significant at the 0.05 level of $P$ value.

be more adaptable to adhere to it [27]. This hypothesis is consistent with our results that follow-up status of the long waiting treatment population is not ideal. Since adherence rate is a key factor to ensure the treatment effects from lots of previous studies $[28,29]$, patients who wait for a long time have higher death rate accordingly. The reduction in waiting time and the number of delayed testers may attribute to the scale-up of the national HIV testing program, free treatment policy, and improved treatment criteria $[14,30]$.

Pretreatment CD4 drop is a precursor of ART treatment outcomes and is associated with a much slower recovery of CD4 level at 6-12 months into treatment. Previous studies have reported that patients with CD4+ T-cell counts less than 200 cells $/ \mathrm{mm}^{3}$ have impaired immunological recovery compared with those start ART while CD4+ T-cell counts above 350 cells $/ \mathrm{mm}^{3}[31,32]$. Our finding also indicated that patients who have a greater CD4 drop may be more likely to discontinue treatment follow-up in the long term. This shows that patients who overlooked own health in waiting duration would be prone to disregard the medical care as well. In clinical settings, doctors can suggest patients with slow CD4 increase test for drug resistance [33]. In the presence of drug resistance, clinical doctors should change the regimen for the patients. Otherwise, doctors should encourage patients to continue treatment to ensure CD4 counts stable, which is still helpful for immune reconstitution and rule out other causes of weak immunological responses $[34,35]$.

Notably, screening of HIV among drug users has reached a high coverage in Yuxi County, but the following HIV treatment comes too late, indicating a weak link in the cascade 
of HIV treatment. This is reflected by the fact that IDU constitutes to a smaller proportion in the delayed group than the nondelayed participants, but with a higher mortality and lossto-follow-up rate during the treatment than heterosexuals. As mentioned in the background, the high-risk population in Yuxi mainly concentrate in IDU. In this situation, local health institute has conducted appropriate prevention for testing to curb the transmission among this group [36, 37]. However, the later follow-up monitoring did not treat IDU as a priority after diagnosis resulting in part of drug users initiate ART late and also a lack of the recommendations from the treatment guidelines was another reason for IDU to start ART late. Thus, specific support is still necessary for the IDU who have HIVpositive confirmed and started ART.

Other independent factors are also associated with mortality or loss-to-follow-up rate. Being enrolled into ART in 2007-2009 led to higher death rate than people started in 2013-2015. It may be because the China-free ART program adopted the optimal first-line ART regimen Tenofovir Disoproxil Fumarate (TDF) instead of Stavudine (d4T) since 2009 [38], and the ART treatment threshold was increased from CD $4 \leq 200$ cells $/ \mathrm{mm}^{3}$ in 2003 to $\leq 350$ cells $/ \mathrm{mm}^{3}$ in 2008 also helps to increase the treatment effects [39]. Elder people have higher mortality and loss-to-follow-up rate; this situation is common in the developing countries [40,41]. As senescence is the nature that human body metabolizes, the function of each organ including immune system has declined with aging which would speed up disease progress.

Several limitations should be noted in this study. First, the patient's health situation of the first CD4+ T-cell test is not collected; the counts would be affected by their infection period or coinfection status. Second, the majority of patients started ART after 2007; this may affect comparability in temporal trend. Third, the study did not capture the detailed information on the reasons when the patients decide to start treatment; this prevents us from excluding patients who delayed the treatment according to the medical suggestions, which leads to selection bias. Fourth, the exact causes of the 26 excluded death cases were not reported by the hospitals to the local CDC. As a result, we cannot independently confirm its relatedness to HIV infection. In this case, HIVrelated mortality may be underestimated. Fifth, there was about $7 \%$ of entry records not being able to be matched across the two databases. One of the likely reason for this is that these individuals may not have local residence such that they were referred back to their home residence for treatment after diagnosis. Hence, they had no treatment data. However, excluding this small proportion of patients is unlikely to alter the study the conclusions.

\section{Conclusion}

Long duration before treatment after HIV-infected confirmation negatively affects the ART effects and adherence rate, which is suggested being shortened. The Chinese treatment guidelines should be updated to account for the needs of pretreatment care and special care for patients coinfected with viral hepatitis. Patients are advised to begin ART when their CD4+ T-cell counts still in the high level. These findings underline the necessary monitoring for HIV-positive patients before treatment to maximize the ART effects in the long term.

\section{Competing Interests}

The authors declare that there are no competing interests.

\section{Authors' Contributions}

Lei Zhang, Jun Jing, Liang Chen, and Feng Cheng conceived and designed the study; Shifu Li, Shunxiang Li, Liangmin Gao, Ying Cai, Jincui Fu, and Chunyuan Guo collected the data; Shifu Li cleaned the data; Shu Su and Lei Zhang analyzed the data; Shu Su wrote the paper; Jun Jing, Limin Mao, and Lei Zhang revised the manuscript. All authors approved the final manuscript.

\section{Acknowledgments}

The authors would like to thank all the patients who participated in this study and grants from Tsinghua University.

\section{References}

[1] J. Poorolajal, E. Hooshmand, H. Mahjub, N. Esmailnasab, and E. Jenabi, "Survival rate of AIDS disease and mortality in HIVinfected patients: a meta-analysis," Public Health, vol. 139, pp. 3-12, 2016.

[2] R. Hammond and T. C. Harry, "Efficacy of antiretroviral therapy in Africa: effect on immunological and virological outcome measures-a meta-analysis," International Journal of STD \& AIDS, vol. 19, no. 5, pp. 291-296, 2008.

[3] J. B. Nachega, O. Adetokunboh, O. A. Uthman et al., "Community-based interventions to improve and sustain antiretroviral therapy adherence, retention in HIV care and clinical outcomes in low- and middle-income countries for achieving the unaids 90-90-90 Targets," Current HIV/AIDS Reports, vol. 13, no. 5, pp. 241-255, 2016.

[4] UNAIDS, 90-90-90: An Ambitious Treatment Target to Help End the AIDS Epidemic, United Nations, 2014.

[5] S. L. Braunstein, M. M. Robertson, J. Myers, and D. Nash, "Using HIV viral load from surveillance to estimate the timing of antiretroviral therapy initiation," Journal of Acquired Immune Deficiency Syndromes, vol. 73, no. 2, pp. 222-227, 2016.

[6] Y. Ding, S. Duan, Z. Wu et al., "Timing of antiretroviral therapy initiation after diagnosis of recent human immunodeficiency virus infection and $\mathrm{CD} 4{ }^{+}$T-cell recovery," Clinical Microbiology and Infection, vol. 22, no. 3, pp. 290.e5-290.e8, 2016.

[7] M. May, M. Gompels, V. Delpech et al., "Impact of late diagnosis and treatment on life expectancy in people with HIV-1: UK Collaborative HIV Cohort (UK CHIC) Study," BMJ (Online), vol. 343, no. 7829, Article ID d6016, 2011.

[8] V. Jain, W. Hartogensis, P. Bacchetti et al., "Antiretroviral therapy initiated within 6 months of HIV infection is associated with lower T-cell activation and smaller HIV reservoir size," Journal of Infectious Diseases, vol. 208, no. 8, pp. 1202-1211, 2013.

[9] WHO, Guideline on When to Start Antiretroviral Therapy and on Pre-Exposure Prophylaxis for HIV, WHO, Geneva, Swizerland, 2015. 
[10] S. P. Eholié, A. Badje, G. M. Kouame et al., "Antiretroviral treatment regardless of CD4 count: the universal answer to a contextual question," AIDS Research and Therapy, vol. 13, article 27, 2016.

[11] H. F. Günthard, M. S. Saag, C. A. Benson et al., "Antiretroviral drugs for treatment and prevention of HIV infection in adults: 2016 recommendations of the international antiviral societyUSA panel," JAMA, vol. 316, no. 2, pp. 191-210, 2016.

[12] Panel on National Free ART Manual, People's Medical Publishing House, Beijing, China, 2008.

[13] J. Wang, Z. Wang, J. Liu et al., "Efficacy and HIV drug resistance profile of second-line ART among patients having received long-term first-line regimens in rural China," Scientific Reports, vol. 5, article 14823, 2015.

[14] L. Zhang, R. T. Gray, and D. P. Wilson, "Modelling the epidemiological impact of scaling up HIV testing and antiretroviral treatment in China," Sexual Health, vol. 9, no. 3, pp. 261-271, 2012.

[15] F. Zhang, J. E. Haberer, Y. Wang et al., "The Chinese free antiretroviral treatment program: challenges and responses," AIDS, vol. 21, no. 8, pp. S143-S148, 2007.

[16] M. Jia, H. Luo, Y. Ma et al., "The HIV epidemic in Yunnan Province, China, 1989-2007," JAIDS Journal of Acquired Immune Deficiency Syndromes, vol. 53, supplement 1, pp. S34-S40, 2010.

[17] M. Chen, L. Yang, Y. Ma et al., "Emerging variability in HIV-1 genetics among recently infected individuals in Yunnan, China," PLoS ONE, vol. 8, no. 3, Article ID e60101, 2013.

[18] S. K. Schwarcz, L. Hsu, C.-S. J. Chin et al., "Do people who develop AIDS within 12 months of HIV diagnosis delay HIV testing?" Public Health Reports, vol. 126, no. 4, pp. 552-559, 2011.

[19] X. Sun, F. Lu, Z. Wu et al., "Evolution of information-driven HIV/AIDS policies in China," International Journal of Epidemiology, vol. 39, supplement 2, pp. ii4-iil3, 2010.

[20] M. Li, W. Tang, K. Bu et al., "Mortality among people living with HIV and AIDS in China: implications for enhancing linkage," Scientific Reports, vol. 6, article 28005, 2016.

[21] R. Bunnell, J. P. Ekwaru, P. Solberg et al., "Changes in sexual behavior and risk of HIV transmission after antiretroviral therapy and prevention interventions in rural Uganda," AIDS, vol. 20, no. 1, pp. 85-92, 2006.

[22] M. S. Cohen, Y. Q. Chen, M. McCauley et al., "Prevention of HIV-1 infection with early antiretroviral therapy," The New England Journal of Medicine, vol. 365, no. 6, pp. 493-505, 2011.

[23] S. M. Abay, K. Deribe, A. A. Reda et al., "The effect of early initiation of antiretroviral therapy in TB/HIV-coinfected patients: a systematic review and meta-analysis," Journal of the International Association of Providers of AIDS Care, vol. 14, no. 6, pp. 560-570, 2015.

[24] M. Chen, W. Wong, M. G. Law et al., "Hepatitis B and C Co-infection in HIV patients from the TREAT Asia HIV observational database: analysis of risk factors and survival," PLOS ONE, vol. 11, no. 3, Article ID e0150512, 2016.

[25] J. L. Marcus, W. A. Leyden, C. R. Chao et al., "Differences in response to antiretroviral therapy by sex and hepatitis $\mathrm{C}$ infection Status," AIDS Patient Care and STDs, vol. 29, no. 7, pp. 370-378, 2015.

[26] L. R. Martin, S. L. Williams, K. B. Haskard, and M. Robin DiMatteo, "The challenge of patient adherence," Therapeutics and Clinical Risk Management, vol. 1, no. 3, pp. 189-199, 2005.

[27] M. K. M. Holstad, J. C. Pace, A. K. De, and D. R. Ura, "Factors associated with adherence to antiretroviral therapy," Journal of the Association of Nurses in AIDS Care, vol. 17, no. 2, pp. 4-15, 2006.

[28] W. M. Bezabhe, L. Chalmers, L. R. Bereznicki, and G. M. Peterson, "Adherence to antiretroviral therapy and virologic failure: a meta-analysis," Medicine, vol. 95, no. 15, 2016.

[29] O. A. Uthman, J. F. Magidson, S. A. Safren, and J. B. Nachega, "Depression and adherence to antiretroviral therapy in low-, middle- and high-income countries: a systematic review and meta-analysis," Current HIV/AIDS reports, vol. 11, no. 3, pp. 291307, 2014.

[30] Z. Wu, Y. Wang, R. Detels, and M. J. Rotheram-Borus, "China AIDS policy implementation: reversing the HIV/AIDS epidemic by 2015," International Journal of Epidemiology, vol. 39, no. 2, Article ID dyq220, pp. iil-ii3, 2010.

[31] S. Su, X. Chen, L. Mao et al., "Superior effects of antiretroviral treatment among men who have sex with men compared to other HIV at-risk populations in a large cohort study in Hunan, China," International Journal of Environmental Research and Public Health, vol. 13, no. 3, p. 283, 2016.

[32] B. Grinsztejn, M. C. Hosseinipour, H. J. Ribaudo et al., "Effects of early versus delayed initiation of antiretroviral treatment on clinical outcomes of HIV-1 infection: results from the phase 3 HPTN 052 randomised controlled trial," The Lancet. Infectious Diseases, vol. 14, no. 4, pp. 281-290, 2014.

[33] S. J. Reynoids, G. Nakigozi, K. Newell et al., "Failure of immunologic criteria to appropriately identify antiretroviral treatment failure in Uganda," AIDS, vol. 23, no. 6, pp. 697-700, 2009.

[34] A. Horta, C. Nobrega, P. Amorim-Machado et al., "Poor immune reconstitution in HIV-infected patients associates with high percentage of regulatory CD4+ T cells," PLOS ONE, vol. 8, no. 2, Article ID e57336, 2013.

[35] H. H. Hirsch, G. Kaufmann, P. Sendi, and M. Battegay, "Immune reconstitution in HIV-infected patients," Clinical Infectious Diseases, vol. 38, no. 8, pp. 1159-1166, 2004.

[36] J. Li and X. Li, "Current status of drug use and HIV/AIDS prevention in drug users in China," Journal of Food and Drug Analysis, vol. 21, no. 4, pp. S37-S41, 2013.

[37] N. Bacaër, X. Abdurahman, and J. Ye, "Modeling the HIV/AIDS epidemic among injecting drug users and sex workers in Kunming, China," Bulletin of Mathematical Biology, vol. 68, no. 3, pp. 525-550, 2006.

[38] Y. Cao, Y. Han, J. Xie et al., "Impact of a tenofovir disoproxil fumarate plus ritonavir-boosted protease inhibitor-based regimen on renal function in HIV-infected individuals: A Prospective, Multicenter Study," BMC Infectious Diseases, vol. 13, no. 1, article 301, 2013.

[39] National Center for AIDS/STD Control and Prevention C.C.N, Panel on National Free ART Manual, People's Medical Publishing House, Beijing, China, 2008.

[40] M. May, A. Boulle, S. Phiri et al., "Prognosis of patients with HIV-1 infection starting antiretroviral therapy in sub-Saharan Africa: a collaborative analysis of scale-up programmes," The Lancet, vol. 376, no. 9739, pp. 449-457, 2010.

[41] A. Boulle, G. Van Cutsem, K. Hilderbrand et al., "Sevenyear experience of a primary care antiretroviral treatment programme in Khayelitsha, South Africa," AIDS, vol. 24, no. 4, pp. 563-572, 2010. 


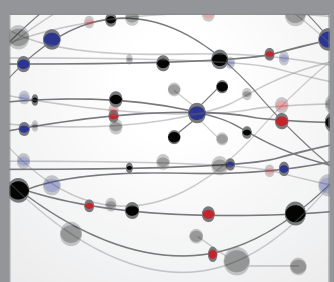

The Scientific World Journal
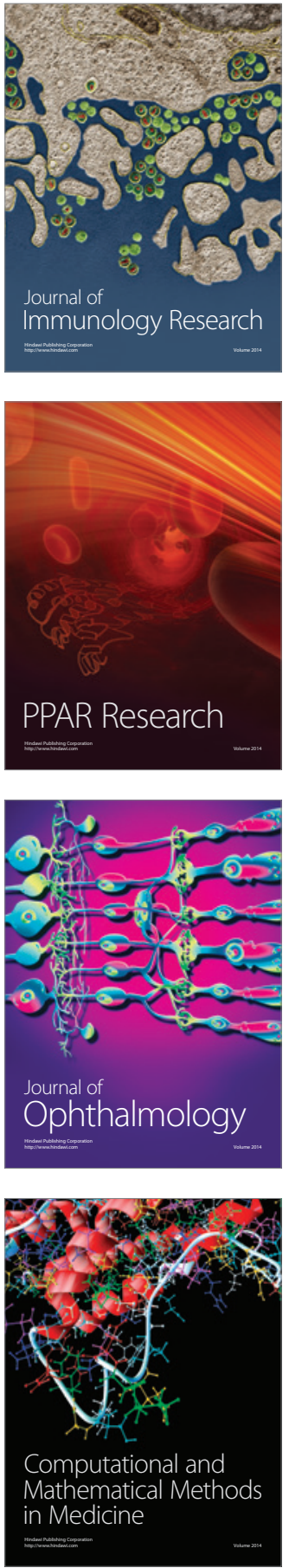

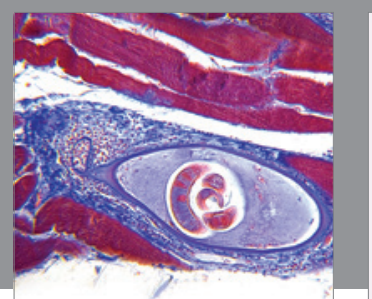

Gastroenterology Research and Practice

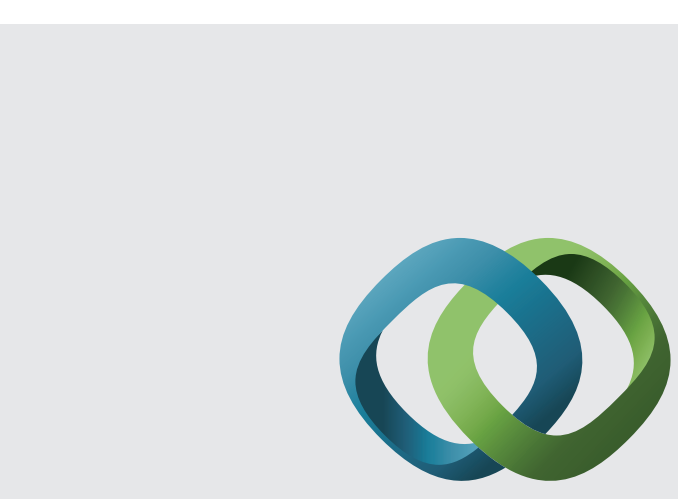

\section{Hindawi}

Submit your manuscripts at

http://www.hindawi.com
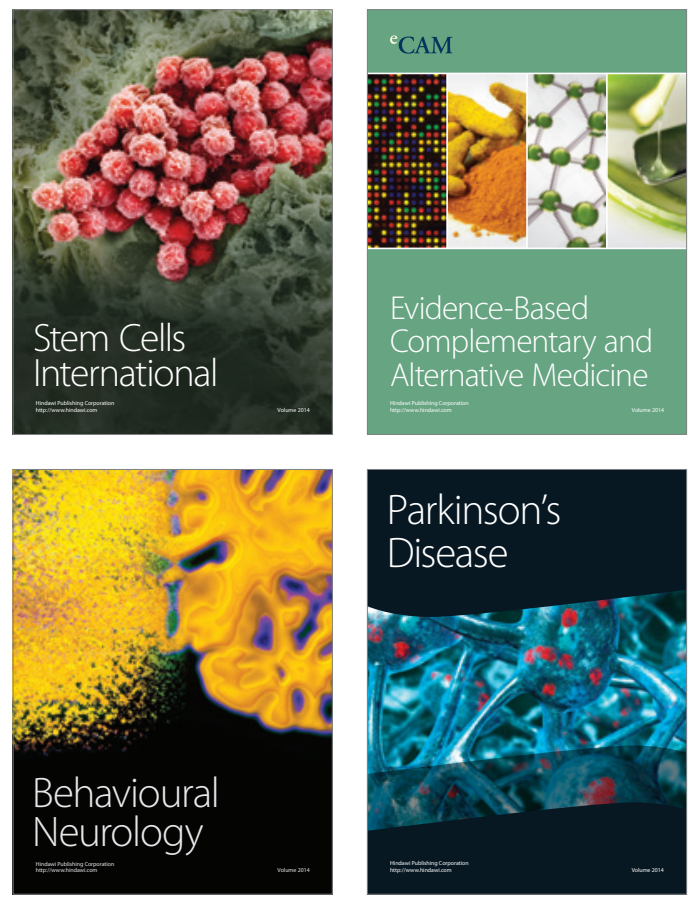
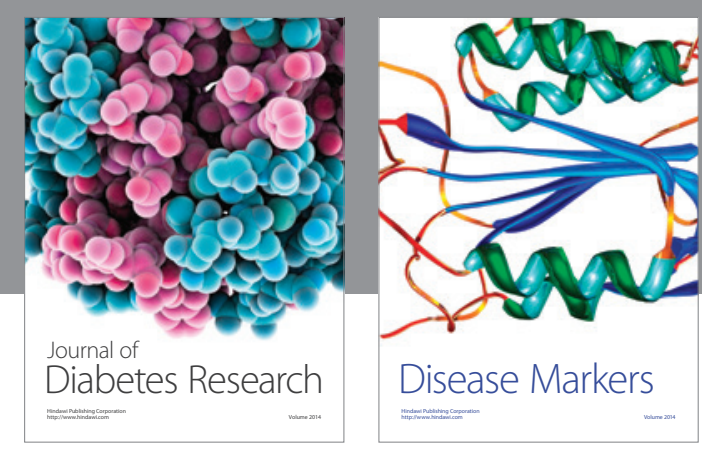

Disease Markers
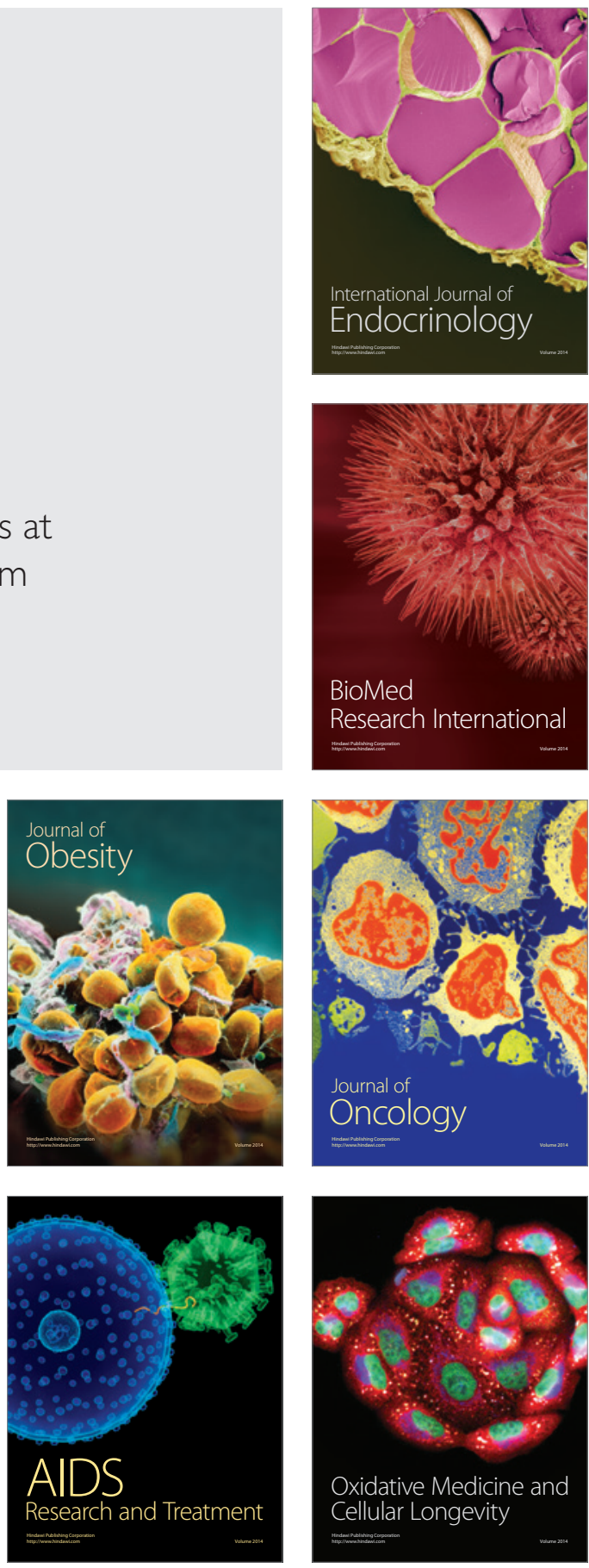